\title{
AN EXAMPLE IN THE THEORY OF WELL-BOUNDED OPERATORS
}

\author{
H. R. DOWSON AND P. G. SPAIN
}

\begin{abstract}
If $H$ is the Hilbert transform on $L^{p}(Z)$, then $T=\pi I+i H$ is a well-bounded operator for $1<p<\infty$, but is not a scalar-type spectral operator except when $p=2$.
\end{abstract}

The purpose of this note is to show that there is a well-bounded operator on a reflexive Banach space which is not scalar-type spectral.

Throughout, let $X$ be a reflexive complex Banach space, and $T$ a bounded linear operator on $X . T$ is said to be well-bounded if and only if it is possible to choose a constant $K$ and a compact interval $J=[a, b]$, such that

$$
\|f(T)\| \leqq K\left\{\sup _{\lambda \in J}|f(\lambda)|+\underset{J}{\operatorname{var} f}\right\}
$$

for every complex polynomial $f$. Such an operator has real spectrum, contained in $J$. Let $\boldsymbol{R}$ denote the set of real numbers. Smart [5, pp. 329-330] and Ringrose [4] have shown that $T$ is well-bounded and satisfies (1) if and only if there is a family $\{E(\lambda): \lambda \in R\}$ of projections on $X$ such that

(i) $\|E(\lambda)\| \leqq K, \lambda \in R$;

(ii) $\lim _{\lambda \rightarrow \mu+} E(\lambda) x=E(\mu) x, x \in X$;

(iii) $\lim _{\lambda \rightarrow \mu_{-}} E(\lambda)$ exists in the strong operator topology;

(iv) $E(\lambda)=0, \lambda<a ; E(\lambda)=I, \lambda \geqq b$;

(v) $T=\int_{J} \lambda d E(\lambda)$,

where the integral exists as a strong limit of Riemann sums.

Now let $S$ be a scalar-type spectral operator on $X$ with real spectrum $\sigma(S)$, contained in the compact interval $J$. It is well known that there is a constant $M$ such that

$$
\|f(S)\| \leqq M \sup _{\lambda \in \sigma(S)}|f(\lambda)| \leqq M \sup _{\lambda \in J}|f(\lambda)|
$$

for every complex polynomial $f$. Moreover, this property characterises the scalar-type spectral operators on $X$ with real spectra [1, Theorem 5.3].

Received by the editors November 13, 1970 and, in revised form, April 26, 1971. AMS 1970 subject classifications. Primary 47B40; Secondary 47A60.

Key words and phrases. Well-bounded operators, scalar-type spectral operators, Hilbert transform.

(c) American Mathematical Society 1972 
Hence every such operator is well-bounded. We show that the converse result fails by proving that the Hilbert transform on $L^{p}(Z)(1<p<\infty)$ determines a well-bounded operator which is not scalar-type spectral unless $p=2$.

Let $\boldsymbol{Z}$ be the locally compact abelian group of integers with (Haar) counting measure. Let $Z^{\wedge}$ be its dual group, the circle, which is isomorphic to the interval $[0,2 \pi]$ with its endpoints identified. Haar measure on $[0,2 \pi]$ is Lebesgue measure divided by $2 \pi$. We denote the norm on $L^{p}(Z)$ by $\|\cdot\|_{p}$.

Let $\tau: L^{2}\left(Z^{\wedge}\right) \rightarrow L^{2}(Z)$ be the Plancherel extension of the Fourier transform, and let ^ be the inverse of $\tau$. If $x, y \in L^{2}\left(Z^{\wedge}\right)$, then $\tau(x y)=\tau(x) * \tau(y)$, where $*$ denotes convolution; also

$$
(\tau x)(n)=\frac{1}{2 \pi} \int_{0}^{2 \pi} x(\lambda) e^{-i n \lambda} d \lambda \quad\left(n \in Z, x \in L\left(Z^{\wedge}\right)\right) .
$$

Let $e_{\theta}=\tau \chi_{[0, \theta]}(0 \leqq \theta \leqq 2 \pi)$. Then $e_{\theta}(0)=\theta / 2 \pi$ and $e_{\theta}(n)=\left(1-e^{-i n \theta}\right) / 2 \pi n i$ $(n \neq 0)$. Thus $e_{\theta} \in L^{p}(Z)(p>1)$ and the set $\left\{e_{\theta}: 0 \leqq \theta \leqq 2 \pi\right\}$ is dominated in $L^{p}(Z)(p>1)$. The identity of the group algebra $L^{1}(Z)$ is $e_{2 \pi}$.

Let $j$ be the function $j:[0,2 \pi] \rightarrow[0,2 \pi]: \lambda \mapsto \lambda$ and let $\delta=\tau j$. Then $\delta(0)=\pi$ and $\delta(n)=i / n(n \neq 0)$. The Hilbert transform $H$ is defined on $L^{p}(Z)(p>1)$ by

(4) $H \xi=\xi *(\cdots,-1 / n, \cdots,-1 / 2,-1,0,1,1 / 2, \cdots, 1 / n, \cdots)$.

Stečkin [6, Corollary 2] has shown that $E(\theta): \xi \mapsto \xi * e_{\theta}(0 \leqq \theta \leqq 2 \pi)$ defines a bounded linear operator on $L^{p}(Z)(p>1)$ and that

$$
\sup \{\|E(\theta)\|: \theta \in[0,2 \pi]\}=K<\infty .
$$

Now let $p$ be fixed in the range $(1,2)$. Let $0 \leqq \theta \leqq \varphi \leqq 2 \pi$, and let $\xi \in L^{p}(Z) \subset$ $L^{2}(Z)$. Then

$$
\begin{aligned}
\left(\xi * e_{\theta}\right) * e_{\varphi} & =\tau\left(\hat{\xi}_{[0, \theta]}\right) * \tau \chi_{[0, \varphi]}=\tau\left(\hat{\xi}_{\left.\chi_{[0, \theta]} \chi_{[0, \varphi]}\right)}\right) \\
& =\xi * e_{\theta}=\left(\xi * e_{\varphi}\right) * e_{\theta} .
\end{aligned}
$$

Hence $\{E(\theta): 0 \leqq \theta \leqq 2 \pi\}$ is a naturally ordered uniformly bounded family of projections on $L^{p}(Z)$.

Since $\left\|e_{\theta}-e_{\varphi}\right\|_{2}=(|\theta-\varphi| / 2 \pi)^{1 / 2}$, we have $\lim _{\varphi \rightarrow \theta} e_{\varphi}(n)=e_{\theta}(n) \quad(n \in Z)$. By the Lebesgue dominated convergence theorem, $\lim _{\varphi \rightarrow \theta}\left\|e_{\varphi}-e_{\theta}\right\|_{p}=0$. For $\xi$ in $L^{p}(Z)$ and $n \geqq 1$ define

$$
\xi_{n}=(\cdots, 0, \xi(-n), \xi(-n+1), \cdots, \xi(n-1), \xi(n), 0, \cdots) .
$$

Then

$$
\begin{aligned}
\left\|\xi *\left(e_{\theta}-e_{\varphi}\right)\right\|_{p} & \leqq\left\|\left(\xi-\xi_{n}\right) *\left(e_{\theta}-e_{\varphi}\right)\right\|_{p}+\left\|\xi_{n} *\left(e_{\theta}-e_{\varphi}\right)\right\|_{p} \\
& \leqq 2 K\left\|\xi-\xi_{n}\right\|_{p}+\left\|\xi_{n}\right\|_{1}\left\|e_{\theta}-e_{\varphi}\right\|_{p}
\end{aligned}
$$


Hence if $0<\theta<2 \pi$,

$$
\begin{aligned}
& E(\theta+)=\lim _{\varphi \rightarrow \theta+} E(\varphi)=E(\theta), \\
& E(\theta-)=\lim _{\varphi \rightarrow \theta-} E(\varphi)=E(\theta), \\
& E(0+)=E(0)=0, \quad E(2 \pi-)=E(2 \pi)=I,
\end{aligned}
$$

the limits being defined in the strong operator topology. Therefore $T=\int_{[0,2 \pi]} \lambda d E(\lambda)$ is a well-bounded operator.

Let $\left\{\lambda_{k}: k=0,1, \cdots, m\right\}$ be a partition of $[0,2 \pi]$, and let $f$ be any complex polynomial. Then, for every $\xi$ in $L^{p}(Z)$,

$$
\sum_{k=1}^{m} f\left(\lambda_{k}\right)\left[E\left(\lambda_{k}\right)-E\left(\lambda_{k-1}\right)\right] \xi=\tau\left\{\sum_{k=1}^{m} f\left(\lambda_{k}\right)\left[\chi_{\left[0, \lambda_{k}\right]}-\chi_{\left[0, \lambda_{k-1}\right]}\right] \hat{\xi}\right\}
$$

Therefore

$$
f(T) \xi=\tau(f \hat{\xi}) .
$$

In particular, $T \xi=\tau(j \hat{\xi})=\delta * \xi$; thus $T=\pi I+i H$.

Now suppose that $T$ is a scalar-type spectral operator, and let $g$ be any complex function continuous on $[0,2 \pi]$. From (3) and (5) we obtain

$$
\left(f(T) e_{2 \pi}\right)(n)=\frac{1}{2 \pi} \int_{0}^{2 \pi} f(\lambda) e^{-i n \lambda} d \lambda
$$

for every complex polynomial $f$. Also $g(T)$ is a bounded linear operator on $L^{p}(Z)$ and from (2), (6) and the Weierstrass polynomial approximation theorem

$$
\left(g(T) e_{2 \pi}\right)(n)=\frac{1}{2 \pi} \int_{0}^{2 \pi} g(\lambda) e^{-i n \lambda} d \lambda .
$$

This gives a contradiction, since not every complex function continuous on $[0,2 \pi]$ has $L^{p}(\boldsymbol{Z})$-summable Fourier coefficients [7, V. 4.11]. We have therefore shown that if $1<p<2$, then $T$ is well-bounded but not scalar-type spectral. Now consider the case $2<p<\infty$, and let $1 / p+1 / q=1$. Then $1<q<2$, and from (4), $H^{*}$ is the Hilbert transform on $L^{q}(Z)$. Therefore $T^{*}$ is well-bounded but not scalar-type spectral. From the characterisations (1) and (2), it follows that $T$ is well-bounded but not scalar-type spectral. We observe at this point that $T$ is not even spectral if $1<p<2$ or $2<p<\infty$ by $[1$, Theorem 5.8]. Finally, if $p=2$, the projections $\{E(\theta): \theta \in[0,2 \pi]\}$ are easily seen to be selfadjoint and so $T$ is selfadjoint. Hence in this case $T$ is both scalar-type spectral and well-bounded.

The operator $T$ has also been discussed in [2, pp. 452-461] and [3, p. 186]. 


\section{REFERENCES}

1. E. Berkson and H. R. Dowson, On uniquely decomposable well-bounded operators, Proc. London Math. Soc. (3) 22 (1971), 339-358.

2. G. L. Krabbe, Convolution operators that satisfy the spectral theorem, Math. Z. 70 (1958/59), 446-462. MR 21 \#2912.

3. G. L. Krabbe, Stieltjes integration, spectral analysis, and the locally-convex algebra (BV), Bull. Amer. Math. Soc. 71 (1965), 184-189. MR 31 \#2631.

4. J. R. Ringrose, On well-bounded operators, J. Austral. Math. Soc. 1 (1959/60), 334-343. MR 23 \#A3463.

5. D. R. Smart, Conditionally convergent spectral expansions, J. Austral. Math. Soc. 1 (1959/60), 319-333. MR 23 \#A3462.

6. S. B. Stečkin, On bilinear forms, Dokl. Akad. Nauk SSSR 71 (1950), 237-240. (Russian) MR 11, 504.

7. A. Zygmund, Trigonometrical series, 2nd rev. ed., Cambridge Univ. Press, New York, 1959. MR 21 \#6498.

Department of Mathematics, The University, Glasgow, W.2, Scotland 